\title{
Repercussões da mastectomia na autoimagem e na vida sexual das mulheres
}

\section{Repercussions of mastectomy on women's self image and sex life}

\section{Repercusiones de la mastectomía em la auto imagen y la vida sexual de las mujeres}

Célio Pereira de Sousa Júnior

ORCID: https://orcid.org/0000-0003-0726-0668 Universidade Federal do Pará, Brasil E-mail: academicocelio@gmail.com

Geísa de Morais Santana

ORCID: https://orcid.org/0000-0001-8008-888X Universidade Estadual do Piauí, Brasil

E-mail: geisasantana97@gmail.com

Antonia Mylene Sousa Almeida

ORCID: https://orcid.org/0000-0003-2695-6505

Faculdade de Educação São Francisco, Brasil

E-mail: mylenesousa123@hotmail.com

Jéssica Fernanda Sousa Serra

ORCID: https://orcid.org/0000-0002-3963-6204

Centro Universitário de Ciências e Tecnologia do Maranhão, Brasil

E-mail: jessica_sousa_97@ @otmail.com

Ana Paula de Carvalho Souza

ORCID: https://orcid.org/0000-0002-4122-6036 Universidade Estadual do Piauí, Brasil E-mail: anapaula.cvlh@gmail.com

Nágila Silva Alves

ORCID: https://orcid.org/0000-0002-1618-8111

Centro Universitário Santo Agostinho, Brasil E-mail: nglarraial@gmail.com

Raiane Lira dos Santos

ORCID: https://orcid.org/0000-0002-8278-4560

Centro Universitário do Estado do Pará, Brasil

E-mail: raianeliradossantos@gmail.com

Jhônata Santos Brito

ORCID: https://orcid.org/0000-0002-8161-5677

Faculdade de Ciências e Empreendedorismo, Brasil

E-mail: jhonbrito12@gmail.com

Andréa Gomes de Almeida

ORCID: https://orcid.org/0000-0002-4221-0562

Centro Universitário de Ciências e Tecnologia do Maranhão, Brasil E-mail: almeidaandrea709@gmail.com

Maria Samara da Silva

ORCID: https://orcid.org/0000-0001-6987-1224 Universidade Estadual do Piauí, Brasil.

E-mail: mariasamara2v@gmail.com

Antonia Aline Rocha de Sousa

ORCID: https://orcid.org/0000-0001-9106-0188

Cristo Faculdade do Piaú, Brasil

E-mail: alinehosha@gmail.com 


\author{
Michelle Quaresma Cardoso \\ ORCID: https://orcid.org/0000-0002-8621-8670 \\ Universidade Federal do Pará, Brasil \\ E-mail: michelle.card91@yahoo.com.br \\ Priscila de Freitas Ferreira \\ ORCID: https://orcid.org/0000-0002-8716-7801 \\ Universidade Federal de Juiz de Fora, Brasil \\ E-mail: prifferreira84@gmail.com \\ Raissa Gabriela de Oliveira Lira \\ ORCID: https://orcid.org/0000-0003-4361-4865 \\ Universidade Norte do Paraná, Brasil \\ E-mail: raissalirafig@gmail.com \\ Alaine dos Santos Silva Martins \\ ORCID: https://orcid.org/0000-0002-1898-4920 \\ Universidade Estadual de Ciências da Saúde de Alagoas, Brasil \\ E-mail: alaine.martins22@gmail.com
}

\begin{abstract}
Resumo
O presente estudo tem como objetivo realizar um levantamento bibliográfico acerca dos impactos da mastectomia na autoimagem e na vida sexual da mulher. Trata-se de uma revisão integrativa da literatura, onde a busca ocorreu através da Biblioteca Virtual em Saúde (BVS), com auxílio das bases de dados Literatura Latino-Americana e do Caribe em Ciências da Saúde (LILACS), Bases de Dados de Enfermagem (BDENF), Scientific Electronic Library Online (SciELO) e MEDLINE. Os artigos foram coletados no período de maio a junho de 2021, sendo utilizados os descritores: Mastectomia, Autoimagem e Mulheres. Após aplicação dos critérios de inclusão, restaram 13 estudos. O presente estudo evidencia que as mamas são consideradas um símbolo para a sexualidade feminina, e que diante do diagnóstico de câncer de mama a mulher vivencia sentimentos negativos como desespero, tristeza, chateação, pavor da morte, incertezas, ansiedade, medo dentre outros. Tais sentimentos estão associados ao câncer como uma doença que engloba diversas negatividades e afeta proporcionalmente a expectativa de continuidade da vida da mulher.
\end{abstract}

Palavras-chave: Mastectomia; Autoimagem; Mulheres.

\begin{abstract}
This study aims to conduct a literature survey about the Impacts of mastectomy on women's self-image and sexual life. This is an integrative literature review, were search occurred in the Virtual Health Library (VHL), with the help of the databases Latin American and Caribbean Literature on health sciences (LILACS), Nursing Database (BDENF), Scientific Eletronic Library Online (SciELO) and PUBMED. The articles were colleted from may to june 2021, being used the descriptors: Mastectomy, Self image and Women. After applying the inclusion criteria, 13 studies remainde. The present study shows that the breasts are considered a symbol for female sexuality, and that when faced with a diagnosis of breast cancer, women experience negative feelings such as despair, sadness, annoyance, fear of death, uncertainty, anxiety, fear, among others. Such feelings are associated with cancer as a disease that encompasses several negativities and proportionally affects the woman's life expectancy.
\end{abstract}

Keywords: Mastectomy; Self image; Women.

\title{
Resumen
}

Este estudio tiene como objetivo realizar un estudio bibliográfico acerca dos impactos de la mastectomía en la autoimagen y la vida sexual de las mujeres. Se trata de una revisión bibliográfica integradora, la búsqueda ocurrió en la Biblioteca Virtual en Salud (BVS), con el auxilio de la Literatura Latin American and Caribbean Literature on Health Sciences (LILACS), Bases de Datos de Enfermería (BDENF), Biblioteca Electrónica Científica en Línea (SciELO) y bases de datos MEDLINE. Los artículos se recogieron de mayo a junio de 2021, siendo utilizado los seguientes descriptores: Mastectomía; Auto imagen e Mujeres. Tras aplicar los criterios de inclusion, quedaron 13 estudios. Este estudio evidencia que las mama son considerada un símbolo de la sexualidad femenina, y que, diante del diagnóstico de cáncer de mama, la mujer experimenta sentimientos negativos como desesperación, tristeza, molestia, miedo a la muerte, incertidumbre, ansiedad, miedo, entre otros. Tales sentimientos están asociados con el cáncer como una doença que engloba diversas negatividades y afecta proporcionalmente la expectativa de vida de la mujer.

Palabras clave: Mastectomía; Auto imagen; Mujeres.

\section{Introdução}

O câncer de mama, assim como outras neoplasias malignas, resulta de uma propagação descontrolada de células anormais, que aparecem em vista de modificações genéticas, sejam elas hereditárias ou obtidas por exposição a fatores ambientais ou fisiológicos. Tais alterações genéticas podem ocasionar mudanças no progresso celular ou na morte celular 
programada, contribuindo para origem do tumor (BRASIL, 2013). Para o Instituto Nacional de Câncer (INCA), no Brasil, estimam-se que surjam 66.280 casos novos de câncer de mama para cada ano do triênio 2020-2022. Esse valor corresponde a um risco estimado de 61,61 casos novos a cada 100 mil mulheres (INCA, 2020).

Os fatores de risco referentes ao câncer de mama estão relacionados à vida reprodutiva da mulher, envelhecimento, histórico familiar de câncer de mama, consumo de álcool, excesso de peso, sedentarismo, exposição à radiação ionizante e alta densidade do tecido mamário (Rocha, Cruz, Vieira, Costa, \& Lima 2016). Além destas, as características reprodutivas estão estabelecidas como um dos fatores de risco devido a doença ser estrogênio-dependente, e compreendem a menarca precoce (aos 11 anos ou menos), a menopausa tardia (aos 55 anos ou mais), a primeira gestação após os 30 anos e a nuliparidade. A influência da amamentação, do uso de contraceptivos e da terapia de reposição hormonal (TRH) após a menopausa ainda são controversas (Silva \& Riul, 2011).

Como tratamento primário, geralmente, realiza-se a intervenção cirúrgica que pode ser restrita ao tumor, atingir tecidos circundantes ou até a retirada total da mama (mastectomia radical) e também a remoção dos linfonodos presentes na região axilar e dos músculos peitorais. Além disso, a mulher pode ser submetida a outros tratamentos como a radioterapia, quimioterapia e hormonioterapia (Hirschle, Maciel \& Amorim, 2018).

Na realização da mastectomia parcial ou cirurgia conservadora, remove-se somente a região da glândula mamária que envolve o tumor, sendo este procedimento constantemente uma alternativa de tratamento para mulheres diagnósticas com câncer de mama em estágio inicial, pois possibilita que ela conserve a maior parte da mama, ainda que evoluem de maneira não muito significativa a taxa de recidiva do local do tumor. Já a mastectomia total, no que lhe diz respeito, é uma intervenção cirúrgica que remove completamente a glândula mamária e os músculos peitorais, com o propósito de diminuir a incidência e melhorar a expectativa de vida das mulheres consideradas de alto risco (Silva, Júnior \& Miranda, 2016).

Após a comprovação do diagnóstico da neoplasia na mama, muitas mulheres vivenciam um momento de imensao sofrimento, angústia e ansiedade, sobretudo pelo motivo de esta ser uma doença estigmatizante na sociedade. Além disso, durante o tratamento elas vivenciam perdas físicas e financeiras, e sintomas adversos como sentimento de perda, depressão, autoimagem prejudicada com diminuição da autoestima, medo de morte e da libido sexual, além de constantes adaptações às mudanças físicas, psicológicas, familiares, sociais e emocionais ocorridas (Lago, Andrade, Nery, \& Avelino, 2015).

A mama é altamente valorizada não só pela pessoa que possui, mas também pela sociedade que, por vezes, a transforma para a composição de uma arte. Por isso que a ausência deste símbolo leva a uma transformação psicológica e funcional do ser humano, pois assim estará interrompendo a realização e o prazer do órgão que acaba sendo uma grande ameaça, já que é a parte do corpo que alimenta não só um bebê, mas também a maternidade e o imaginário social sobre o feminino. Em relação à imagem corporal, as mulheres que são submetidas a cirurgia tipo mastectomia radical se deparam com uma ameaça a sua integridade corporal juntamente com a sua satisfação, pois tem uma visão do seu corpo como algo intacto, completo e funcionando inteiramente. A imagem corporal após mastectomia é ameaçadora, porque a paciente irá conviver com uma mutilação (Prates, Zanini \& Veloso, 2012).

É importante salientar que a neoplasia mamária requer tratamentos que levam à alteração da autoimagem e da autoestima da mulher, especialmente as modalidades cirúrgica e quimioterápica, por causarem a perda total ou parcial da mama e a queda dos cabelos e pelos do corpo, fatores que podem interferir na sexualidade e levar a mulher à dificuldade de se relacionar sexualmente com seu companheiro (Mairink, Gradim, Gozzo, Fendrich, \& Panobianco, 2020).

Cabe ao profissional de saúde tentar clarificar as questões que as mulheres têm nos mais variados atendimentos, já que as dificuldades em viver a sexualidade são mais comuns do que se pode imaginar. Entretanto, a ausência de abertura na assistência impossibilita que se forme um vínculo entre paciente oncológico e profissional, o que torna difícil a verbalização do problema (Cesnik \& Santos, 2012). 
Frente ao exposto, o presente trabalho justifica-se pela necessidade de traçar os impactos causados pela mastectomia na autoimagem e na sexualidade da mulher, além de descrever os sentimentos vividos por elas desde o diagnóstico do câncer mamária, a mastectomia e o pós-cirúrgico. O conhecimento atribuído pelo exame do impacto físico desencadeado na mulher acometida pelo câncer de mama pode favorecer com uma melhor formação, sensibilização e instrumentalização dos profissionais da área de saúde em relação ao tema e, assim, promover uma assistência qualificada a essas mulheres que necessitam de suporte.

O presente estudo tem como objetivo realizar um levantamento bibliográfico acerca dos impactos da mastectomia na autoimagem e na vida sexual da mulher.

\section{Metodologia}

O presente estudo trata-se de um estudo descritivo, do tipo revisão integrativa da literatura, de caráter qualitativo, visto que é definida como um tipo de investigação voltada para o aspecto qualitativo de uma determinada questão, nesse caso, os impactos da mastectomia na autoimagem e na sexualidade da mulher. A revisão de literatura permite aprofundar dentro de diversos autores e referenciais, sobre os discursos e principais temas abordados (Pereira et al., 2018).

Para a revisão integrativa da literatura foram percorridas as seguintes etapas: identificação do tema e seleção da questão norteadora, estabelecimento de critérios de inclusão e exclusão de estudos, categorização e avaliação dos artigos incluídos na revisão, interpretação dos resultados e a síntese do conhecimento dos principais resultados evidenciados na análise dos artigos incluídos.

O tema, determinou a construção da estratégia PICo, que representa uma acrômio para Paciente ou Problema (P), Interesse (I) e Contexto (Co), na qual foi utilizada para a geração da questão norteadora desta revisão integrativa da literatura: “Quais os impactos que a mastectomia causa na autoimagem e na vida sexual da mulher?”.

O levantamento do estudo foi realizado entre maio e junho de 2021, onde a busca dos artigos foi realizada através da Biblioteca Virtual em Saúde (BVS), com auxílio das bases de dados Literatura Latino-Americana e do Caribe em Ciências da Saúde (LILACS), Base de Dados de Enfermagem (BDENF), Scientific Electronic Library Online (SciELO) e PUBMED.

Para a localização dos estudos relevantes, que respondessem à pergunta da pesquisa, utilizou-se os descritores: "Mastectomia”, “Autoimagem” e "Mulheres”, localizados na lista dos Descritores em Ciências da Saúde (DeCS), disponíveis no portal da Biblioteca Virtual em Saúde em (http://decs.bvs.br/). Tais descritores foram cruzados utilizando os operadores booleanos “AND” para obtenção dos critérios de inclusão e exclusão.

Os critérios de inclusão estabelecidos para a seleção dos artigos foram: artigos completos publicados nas bases de dados elencadas, nos idiomas português e inglês, com recorte temporal de 2012 a 2021 e relacionados com a temática. Foram excluídos os estudos duplicados, incompletos, cartas ao editor, resumos, resenhas, monografias, dissertações, teses, e artigos publicados em anais de eventos, dentre aqueles que estão fora do recorte temporal.

\section{Resultados e Discussão}

A partir da revisão de literatura e análise dos estudos indexados nas bases de dados eletrônicas, acerca da temática proposta, foram identificados inicialmente 62 artigos científicos, sendo que apenas 32 foram selecionados. Destes, 18 atenderam aos critérios de inclusão previamente estabelecidos, sendo que 05 foram excluídos de acordo com os critérios de exclusão, resultando apenas 13 artigos para análise e composição do presente estudo. O fluxograma como o detalhamento das etapas da pesquisa está apresentado a seguir na Figura 1. 
Figura 1. Fluxograma de identificação e seleção dos estudos, 2021.
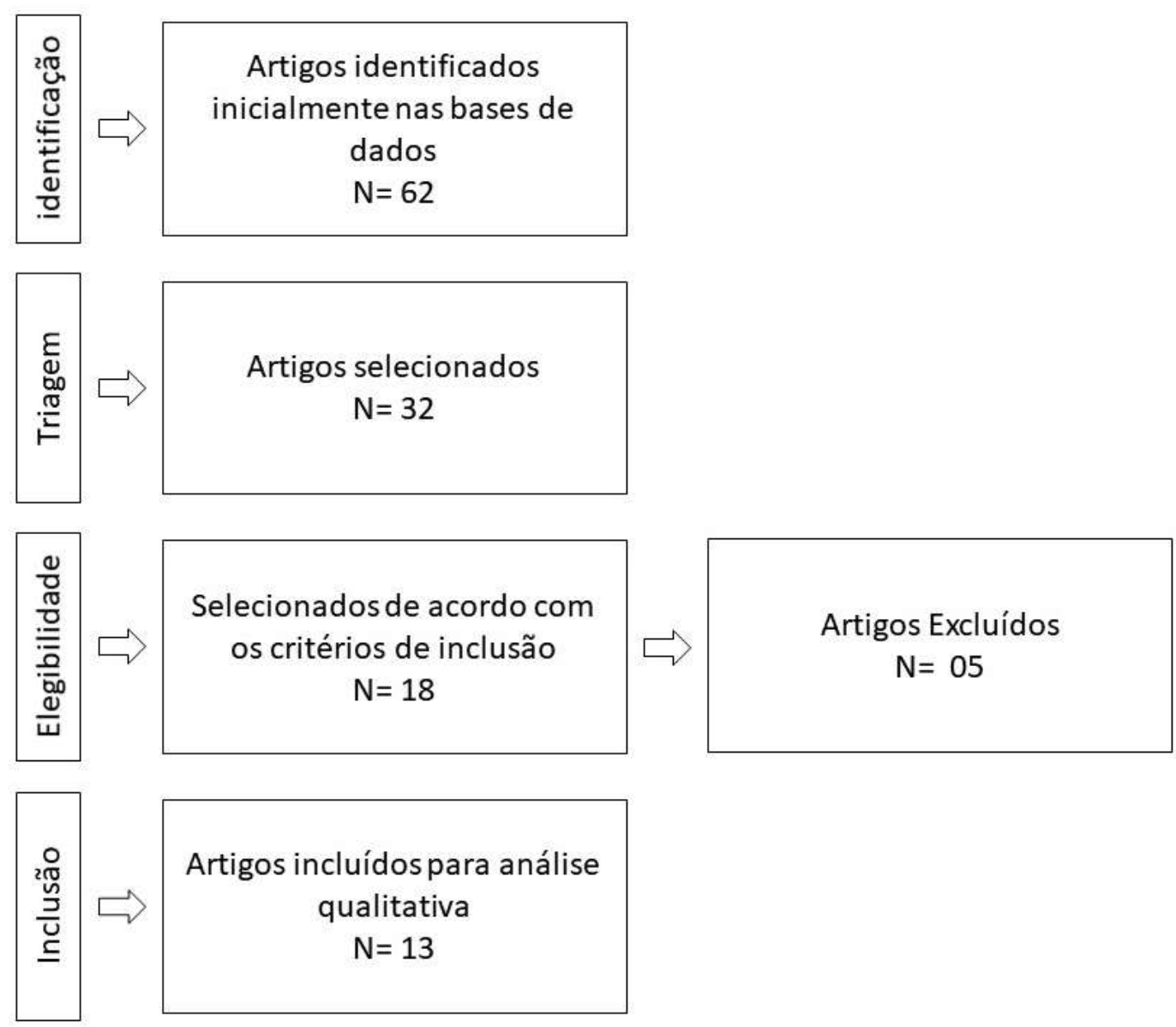

Fonte: Autores (2021).

Os estudos 13 artigos selecionados para a composição da presente revisão integrativa da literatura estão apresentados no Quadro 1 segundo título do artigo, autores e ano de publicação, objetivos, metodologia e principais resultados.

Quadro 1. Publicações incluídas segundo título do artigo, autores e ano de publicação, objetivos, metodologia e principais resultados.

\begin{tabular}{|c|c|c|c|c|}
\hline Título & Autores / ano & Objetivo principal & Metodologia & Principais resultados \\
\hline $\begin{array}{l}\text { Cuidado de enfermagem } \\
\text { na perspectiva do mundo } \\
\text { da vida da mulher-que- } \\
\text { vivencia-linfedema- } \\
\text { decorrente-do- } \\
\text { tratamento-de-câncer-de- } \\
\text { mama. }\end{array}$ & $\begin{array}{l}\text { Paiva, Elias e } \\
\text { Souza } \\
(2020)\end{array}$ & $\begin{array}{l}\text { Desvelar sentidos da mulher } \\
\text { na vivência do linfedema } \\
\text { decorrente do tratamento de } \\
\text { câncer de mama e analisar } \\
\text { propostas de cuidado na } \\
\text { perspectiva do mundo da } \\
\text { vida dessa mulher. }\end{array}$ & $\begin{array}{c}\text { Estudo qualitativo de } \\
\text { abordagem } \\
\text { fenomenológica }\end{array}$ & $\begin{array}{l}\text { As mulheres mostraram-se envergonhadas e } \\
\text { chateadas com o braço sem estética. Ficam } \\
\text { deprimidas, perdem a autoestima e tentam } \\
\text { disfarçar, mas nem sempre é possível. Em } \\
\text { alguns momentos, preferem não sair de casa. } \\
\text { Sentem dificuldade de comprar roupas que } \\
\text { se ajustem no braço edemaciado. }\end{array}$ \\
\hline $\begin{array}{l}\text { Vivência de mulheres } \\
\text { jovens diante da } \\
\text { neoplasia mamaria. }\end{array}$ & $\begin{array}{l}\text { Mairink, Gradim, } \\
\text { Gozzo, Canete, } \\
\text { Fendrich e } \\
\text { Panobianco } \\
\text { (2020) }\end{array}$ & $\begin{array}{c}\text { Compreender a vivência de } \\
\text { mulheres } \\
\text { jovens (18 a } 40 \text { anos) em } \\
\text { tratamento da neoplasia } \\
\text { mamária. }\end{array}$ & $\begin{array}{l}\text { Estudo qualitativo, } \\
\text { com referenciais } \\
\text { teórico e } \\
\text { metodológico: } \\
\text { interacionismo } \\
\text { simbólico e teoria } \\
\text { fundamentada nos } \\
\text { dados }\end{array}$ & $\begin{array}{l}\text { Destaca-se que a perda da vaidade no grupo } \\
\text { jovem estudado foi o ponto mais marcante e } \\
\text { veio associado à alopecia, à mastectomia e } \\
\text { ao déficit de autonomia, com prejuízo de } \\
\text { atividades laborais }\end{array}$ \\
\hline
\end{tabular}




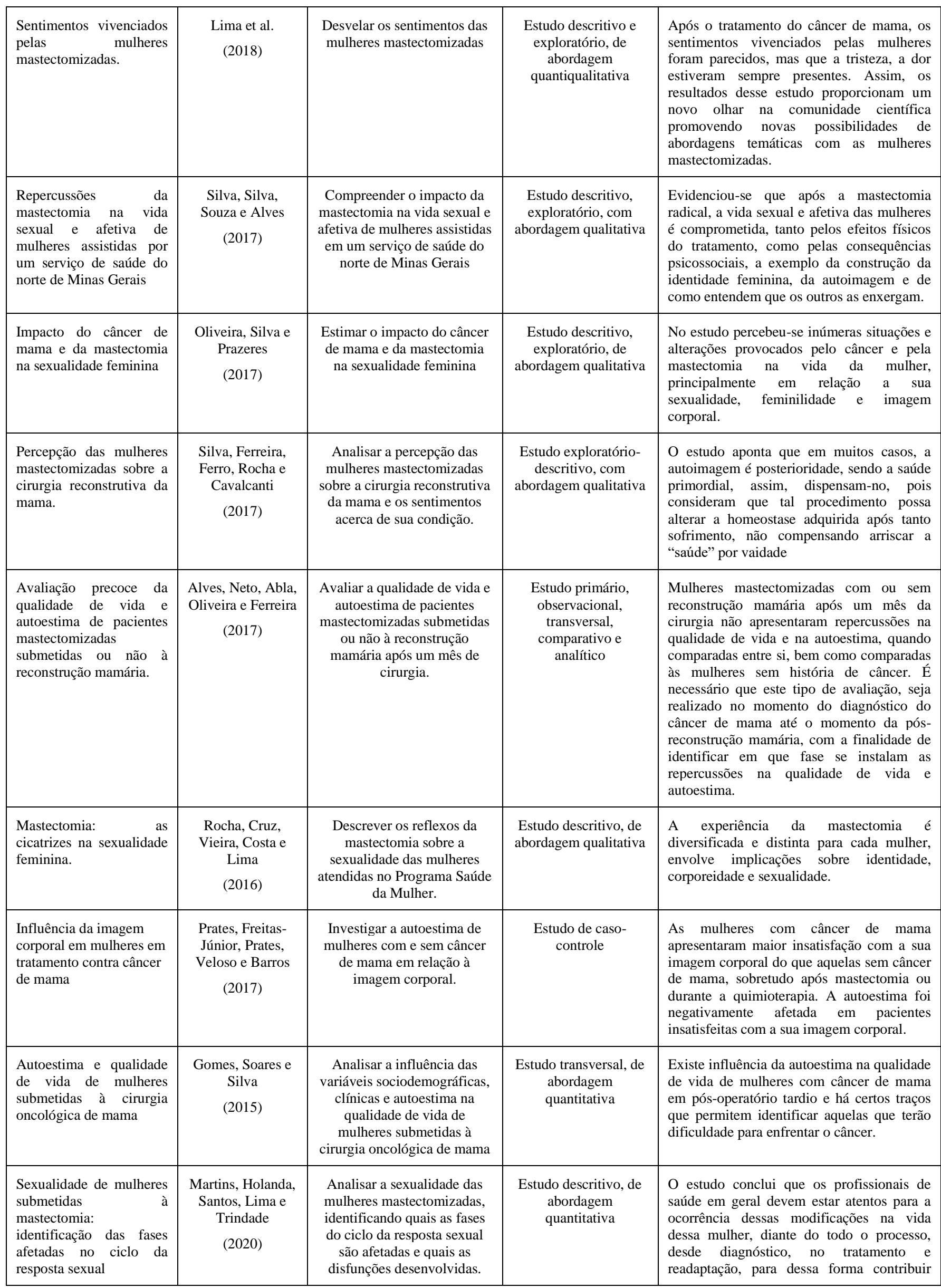




\begin{tabular}{|l|c|c|c|l|}
\hline $\begin{array}{l}\text { Autoestima de mulheres } \\
\text { mastectomizadas } \\
\text { aplicação da escala de } \\
\text { Rosenberg }\end{array}$ & $\begin{array}{c}\text { Fernandes, Alves, } \\
\text { Santos, Mota e } \\
\text { Fernandes } \\
\text { (2013) }\end{array}$ & $\begin{array}{l}\text { Avaliar o nível de autoestima } \\
\text { de mulheres mastectomizadas } \\
\text { com a aplicação da Escala de } \\
\text { Autoestima de Rosenberg }\end{array}$ & $\begin{array}{c}\text { Estudo transversal, do } \\
\text { tipo exploratório- } \\
\text { descritivo com } \\
\text { abordagem } \\
\text { quantitativa }\end{array}$ & $\begin{array}{l}\text { A avaliação da autoestima de mulheres } \\
\text { mastectomizadas pode auxiliar na adoção de } \\
\text { intervenções de enfermagem capazes de } \\
\text { provocar mudanças no tipo de cuidado } \\
\text { prestado a essa clientela. }\end{array}$ \\
\hline $\begin{array}{l}\text { Análise da qualidade de } \\
\text { vida em mulhes } \\
\text { mastectomizadas } \\
\text { atendidas no ambulatório } \\
\text { de HBDF }\end{array}$ & $\begin{array}{c}\text { Sousa, Sant’Ana e } \\
\text { Costa }\end{array}$ & $\begin{array}{c}\text { Avaliar os principais fatores } \\
\text { que influenciam na qualidade } \\
\text { de vida em mulheres } \\
\text { mastectomizadas e atendidas } \\
\text { no ambulatório do Hospital } \\
\text { de Base do Distrito Federal } \\
\text { (HBDF) e identificar os } \\
\text { diagnósticos de enfermagem } \\
\text { que mais prevalecerem. }\end{array}$ & $\begin{array}{c}\text { Estudo descritivo, com } \\
\text { abordagem } \\
\text { quantitativa }\end{array}$ & $\begin{array}{l}\text { O estudo observou uma boa qualidade de } \\
\text { vida nas mulheres avaliadas, sendo o } \\
\text { domínio físico o mais prejudicado e o } \\
\text { psicológico o mais preservado. Espera-se } \\
\text { que por meio de uma assistência } \\
\text { fundamentada no processo de enfermagem } \\
\text { possa promover uma melhora da qualidade } \\
\text { de vida dessas mulheres }\end{array}$ \\
\hline
\end{tabular}

Fonte: Autores (2021).

O câncer de mama pode desorganizar diversos aspectos na vida da mulher, seja ele físico, social e emocional, o que vai depender da gravidade, dos imprevistos no curso da doença, das mudanças na estrutura corporal e na alteração da autoimagem (Paiva, Elias, \& Souza, 2020). A descoberta da doença leva ao medo da morte e a mulher emocionalmente, fazendo-a repensar seu modo de viver, redefinindo suas prioridades e voltando a atenção para o seu eu (Mairink et al., 2020).

Lima et al. (2018) cita em seu estudo que as emoções que as mulheres vivenciam com câncer, a partir da aceitação do diagnóstico até a realização do seu tratamento, tem grande influencia no processo saúde/doença. Sentimentos como o desespero, a raiva, inquietação, tristeza, angústia, ansiedade, medo e luto são considerados comuns no decorrer deste processo, apesar que cada paciente vivencia de maneira individual cada fase.

O diagnóstico do câncer de mama aliada a mastectomia resultam em uma experiência repleta de temor para as mulheres, pois a maior parte delas quando recebe o diagnóstico sente-se angustiada, insegura e preocupada com o desenvolvimento da doença em seu corpo e o tratamento, bem como com os efeitos colaterais e a possibilidade ou não de sobrevida. Em vista disso, predominam sentimentos negativos como o medo do desconhecido e da morte, rejeição, culta e a perda do sentido da vida associado a mudanças em decorrência do processo cirúrgico (Silva, Silva, Souza, \& Alves, 2017).

Oliveira, Silva e Prazeres (2017) citam que o sofrimento psíquico acompanha as pacientes desde o diagnóstico e que podendo variar de intensidade, conforme o tempo e a capacidade individual de cada mulher acometida pelo câncer de mama. A confirmação diagnóstica favorece a sensação de incertezas quanto à vida futura, com pavor da morte. Citam ainda que a descrição do momento de informação do diagnóstico é reveladora de experiências marcantes para as mulheres e que diante da descoberta do câncer de mama, o susto e o desespero o medo da morte são os sentimentos mais relatados entre as mulheres, deixando-se manifestar através do choro.

Com o fato de saber que irá realizar a mastectomia, a mulher sente-se fragilizada, e a retirada da mama torna-se um processo difícil para a aceitação, insinuando que as experiências vivenciadas neste momento são bastante significativas, aumentando assim o sofrimento (Silva, Ferreira, Ferro, Rocha, \& Cavalcanti, 2017).

A mama é considerada importante para a construção da autoimagem. A sensação de estar completa traz a mulher autoestima e boa aceitação da sua imagem corporal (Inocenti, Santos, Loyola, Magalhães, \& Panobianco, 2016). No entanto, imagem corporal tem sido muito valorizada na sociedade e principalmente nos meios de comunicação em geral, refletindo de forma considerável na vida das pessoas, principalmente das mulheres. Portanto, após o procedimento cirúrgico, ratificam a insatisfação do parceiro com a perda da mama, gerando sentimentos de tristeza e desvalorização da imagem feminina, destacando ainda o medo de não ser mais atraente sexualmente e a sensação de diminuição da feminilidade, acarretando prejuízo da autoestima (Rocha, Cruz, Vieira, Costa, \& Lima, 2016). 
De acordo com Alves, Neto, Abla, Oliveira e Ferreira (2017) a sensação de integridade corporal é essencial para o ser humano, e a mastectomia conduz à perda de parte da capacidade de executar tarefas diárias e da autoestima, contribuindo para o declínio na qualidade de vida. A autoestima relaciona-se diretamente às questões psicológicas e físicas, confirmando a importância de ter sentimentos positivos em relação a si mesmo nas diversas experiências vividas. Além disso, a autoestima elevada, durante o período de diagnóstico, tratamento e acompanhamento do câncer de mama, poderia influenciar positivamente a qualidade de vida dessas pacientes, favorecendo seu bem-estar físico, psíquico e emocional.

As mamas são sentidas e vistas como um símbolo do ser feminino, estando ligadas ao erotismo, à sexualidade, como também à função da amamentação e a sua extinção e adoecimento afetam a sexualidade e corporeidade feminina, sendo inegável a supervalorização do corpo nos tempos atuais (Rocha, Cruz, Vieira, Costa, \& Lima, 2016).

Ser submetida à mastectomia resulta em um efeito negativo em relação à aparência, sendo entendido como um grande desafio para as mulheres, exercendo sentimentos negativos como aspectos emocionais e sociais. Neste sentido, a preocupação com a imagem corporal é bem mais elevada em pacientes jovens com câncer de mama, descrevendo à mastectomia como uma mutilação terrível, que é emocionalmente devastadora e as leva a evitar interações sociais e intimidade física, o que as leva a se sentirem-se inadequadas em relação à feminilidade e tendo dificuldade de aceitar o novo corpo (Prates, Freitas-Júnior, Prates, Veloso, \& Barros, 2017).

Para a recuperação psíquica e sexual, o companheiro afetivo e/ou sexual adquire grande importância. O apoio emocional e sincero deste faz com que o conflito da aceitação do procedimento cirúrgico seja encarado mais facilmente, assim como a continuidade da união. O companheiro da mulher com câncer de mama também apresenta sentimentos ambíguos, como medo, tristeza, esperança, fé e alegria, de acordo com cada etapa vivenciada. Assim, a criação de estratégias de suporte para ele é necessária, na tentativa de manter uma base familiar sólida, o que refletirá diretamente na autoestima e qualidade de vida dessas mulheres (Gomes, Soares, \& Silva, 2015).

Pesquisas evidenciam que o apoio do companheiro influencia bastante na vivência da sexualidade da mulher que adoece com câncer de mama em todas as etapas da doença, necessitando de compreensão e dedicação para sentir-se mais segura e lidar com a situação. Sendo apontado ainda as vantagens da existência de um bom relacionamento conjugal e a presença de satisfação sexual, concluindo que, quando a qualidade do relacionamento conjugal é boa, diminui-se a insatisfação sexual (Martins, Holanda, Santos, Lima, \& Trindade, 2020)

Fernandes, Alves, Santos, Mota e Fernandes (2013) pontuam ainda que após a retirada da mama, a mulher deve buscar estratégias que propiciem mudanças de comportamento no seu estilo de vida e nas formas de pensar e refletir sobre a doença, destacando-se que o corpo e a mente devem estar em harmonia para a procura por um equilíbrio emocional, motivando-as a preservar a saúde e o estado de autoestima. Além disso, a participação das mulheres mastectomizadas em grupos de apoio é de importância fundamental, pois possibilita a troca de experiências, oferece suporte social, de informações e orientações sobre a doença e seus tratamentos, além do desenvolvimento de atividades de lazer, melhorando a autoimagem, autoestima e comunicação interpessoal.

Apesar do impacto que uma doença como o câncer de mama causa nas pessoas, o apoio da família, a presença de relações afetuosas, uma maior segurança na união, traz um melhor enfrentamento do problema. Desta forma, o apoio conjugal e familiar faz-se necessário e é salutar para o enfrentamento da doença, já que o homem e a mulher se ajudam mutualmente, amenizando os flagelos decorrentes da enfermidade (Silva, Silva, Souza, \& Alves, 2017).

Em vista disso, cabe também ao profissional de saúde prestar uma assistência que favoreça uma melhora da qualidade de vida, atuando tanto no aspecto psicossocial como na readaptação do estilo de vida dessas mulheres. Sendo assim a assistência prestada às mulheres são fundamentais para o processo saúde-doença. Nesse aspecto, os profissionais de saúde, sobretudo os de enfermagem são fundamentais no auxílio dessas mulheres no que tange os aspectos físicos, psicológicos, 
sociais e a interação com meio ambiente visando uma assistência individualizada fundamentados no processo de enfermagem (Sousa, Sant'Ana, \& Costa, 2014).

\section{Considerações Finais}

O presente estudo evidencia que as mamas são consideradas um símbolo para a sexualidade feminina, e que diante do diagnóstico de câncer de mama a mulher vivencia sentimentos negativos como desespero, tristeza, chateação, pavor da morte, incertezas, ansiedade, medo dentre outros. Tais sentimentos associam-se ao câncer como uma doença que abarca variadas negatividades e que afeta proporcionalmente a expectativa para a continuidade da vida.

Por tanto, as mulheres necessitam ser encorajadas a entender e aceitar-se após a mastectomia, evitando que os sentimentos negativos advindos dos impactos do câncer acarretem sua autoestima, autoestima e o seu psicológico. Para isso torna-se essencial o apoio do parceiro conjugal, da família e dos amigos no enfrentamento a perda mamária da mulher, torna-a um ser emponderada e sem sentimentos de exclusão social.

Conclui-se que esta revisão integrativa é relevante para a literatura, pois permite o incentivo do reconhecimento e da importância de uma assistência holística e humanizada a mulher diagnosticada com câncer de mama e submetidas a mastectomia. Desta forma, os profissionais de saúde devem desenvolver de uma assistência adequada à mulher mastectomizada, proporcionando maior tranquilidade e conforto, com uma participação ativa dos profissionais de enfermagem junto à mulher e seus familiares no processo de recuperação e reabilitação, facilitando o cuidado integral, e auxiliando na minimização dos danos desenvolvidos por todo o processo do tratamento.

Diante do contexto apresentado nesta revisão integrativa da literatura, foi possível identificar que o profissional de saúde deve se aprimorar seu conhecimento e focar na mulher como um todo. De acordo com os estudos selecionados as questões relacionadas à sexualidade e a autoimagem são fundamentais para a mulher, porém pouco trabalhadas, uma vez que o foco principal se dá entorno da doença.

Sugere-se que futuros estudos abordem a assistência que os profissional de saúde prestam à mulher mastectomizada, visando aprimorar a prática do cuidado e as atividades realizadas a população feminina, contribuindo para uma assistência humanizada, integral e acolhedora à mulher diante dos impactos causados pelo diagnóstico e tratamento da mastectomia.

\section{Referências}

Alves, V. L., Neto, M. S., Abla, L. E. F., Oliveira, C. J. R., \& Ferreira, L. M. (2017). Avaliação precoce da qualidade de vida e autoestima de pacientes mastectomizadas submetidas ou não à reconstrução mamária. Rev. Bras. De Cir. Plást., 32(2), 208-217.

Brasil. (2013). Controle dos cânceres do colo do útero e da mama / Ministério da Saúde, Secretaria de Atenção à Saúde, Departamento de Atenção Básica. - 2. ed. - Brasília: Editora do Ministério da Saúde.

Cesnik, V. M., \& Santos, M. A., (2012). Desconfortos físicos decorrentes dos tratamentos do câncer de mama influenciam a sexualidade da mulher mastectomizada. Revista da Escola de Enfermagem USP, 46(4),1001-8.

Fernandes, M. M. J., Alves, C., Santos, M. C. L., Mota, E. M., \& Fernandes, A. F. C. (2013). Autoestima de mulheres mastectomizadas - aplicação da escala de rosenberg. Rev. Rene, Fortaleza, 14(1), p.101-108.

Gomes, N. S., Soares, M. B. O., \& Silva, S. R. (2015). Autoestima e qualidade de vida de mulheres submetidas à cirurgia oncológica de mama. Rev Min Enferm. 19(2): 120-126.

Hirschle, T. M. R., Maciel, S. C., \& Amorim, G. K. (2018). Representações Sociais sobre o Corpo e Satisfação Sexual de Mulheres Mastectomizadas e seus Parceiros. Trends in Psychology. 26(1), 457-468.

Instituto Nacional de Câncer José Alencar Gomes da Silva. (2020). Estimativa 2018: incidência de câncer no Brasil. Coordenação de Prevenção e Vigilância. - Rio de Janeiro: INCA.

Lago, E. A., Andrade, N. K. S., Nery, I. S., \& Avelino, F. V. S. D. (2015). Sentimento de mulheres mastectomizadas acerca da autoimagem e alterações na vida diária. Ciência \& Saúde. (1):15-18. 
Lima, M. M. G., et al. (2018). Sentimentos vivenciados pelas mulheres mastectomizadas. Rev enferm UFPE on line., Recife, 12(5):1216-24.

Mairink, A. A. R., Gradim, C. V. C., Gozzo, T. O., Canete, A. C. S., Fendrich, L., \& Panobianco, M. S. (2020). A prática sexual de mulheres jovens em tratamento para o câncer de mama. Esc Anna Nery. 24(3):e20190360.

Martins, J. O. A., Holanda, J. B. L., Santos, A. A. P., Lima, L. K. P., \& Trindade, R. F. C. (2020). Sexualidade de mulheres submetidas à mastectomia: identificação das fases afetadas no ciclo da resposta sexual. R. pesq.: cuid. fundam., 12: 67-72.

Oliveira, F. B. M., Silva, F. S., \& Prazeres, A. S. B. (2018). Impacto do câncer de mama e da mastectomia na sexualidade feminina. Rev enferm UFPE on line., Recife, 11(Supl. 6): 2533-2540

Paiva, A. C. C., Elias, E. A., Souza, I. E. O., Moreira, M. C., Melo, M. C. S. C., \& Amorim, T. V. (2020). Cuidado de enfermagem na perspectiva do mundo da vida da mulher-que-vivencia-linfedema-decorrente-do-tratamento-de-câncer-de-mama. Esc. Anna Nery, $24(2)$ : e20190176.

Pereira, A. S. (2018). Metodologia da pesquisa científica. UFSM. https://repositorio.ufsm.br/bitstream/handle/1/15824/Lic_Computacao_MetodologiaPesquisa-Cientifica.pdf?sequence $=1$.

Prates, A. C. L., Freitas-Júnior, R., Prates, M. F. O., Veloso, M. F., \& Barros, N. M. (2017). Influência da imagem corporal em mulheres submetidas a tratamento de câncer de mama. Revista Brasileira de Ginecologia e Obstetrícia, 39(4): 175-183.

Prates, A. C. L., Zanini, D. S., \& Veloso, M. F. (2012). Investimento corporal e o funcionamento sexual em mulheres no pós-cirúrgico de câncer de mama. Rev. SBPH, Rio de Janeiro, 15(1), 264-278.

Rocha, J. F. D., Cruz, K. R., Vieira, M. A., Costa, F. M., \& Lima, C. A. (2016). Mastectomia: as cicatrizes da sexualidade masculina. Rev enferm UFPE on line., Recife, 10(Supl. 5):4255-63.

Silva, A., \& Riul, S. S. (2011). Câncer de mama: fatores de risco e detecção precoce. Revista Brasileira de Enfermagem, 64(6), 1016-1021.

Silva, M. B., Júnior, J. M. P., \& Miranda, F. A. N. (2016). Life trajectory of mastectomized women based on the collective subject discourse. Rev. de pesquisa Cuidado é Fundamental. 8(2):4365-75.

Sousa, A. L. V., Sant'Ana, G., \& Costa, Z. M. B. (2014). Análise da qualidade de vida em mulheres mastectomizadas atendidas no ambulatório de HBDF. Com. Ciências Saúde. 25(1): 13-24. 